\title{
A FIXED-POINT THEOREM FOR INWARD AND OUTWARD MAPS
}

\author{
BY \\ BENJAMIN R. HALPERN AND GEORGE M. BERGMAN( ${ }^{(1)}$
}

The Schauder-Tychonoff theorem states that a continuous function from a compact convex subset of a locally convex topological vector space into itself must have a fixed point ([1, Chapter V, 10.5], or [2]). Using this theorem, we obtain here a stronger result, stating that a map from such a set into the surrounding vector space has a fixed point if the directions in which the points are moved satisfy a certain "inwardness" condition.

It follows immediately that a symmetrical "outwardness" condition also implies the existence of a fixed point. We find also that under the latter condition the image of the map necessarily includes the original set!

1. Definitions. Let $X$ be a topological vector space, and $K$ a compact convex subset of $X$.

We shall call a map $F: K \rightarrow X$ "inner" if $F(K) \subset K$.

Given $x \in K$, let us define the "inward set" of $x$ with respect to $K$ as the set of points of the form $(1-\alpha) x+\alpha y$, for $y \in K, \alpha \geqq 0$. It can be thought of as the union of all rays originating at $x$ and drawn so as to pass through some other point $y$ of $K$. For $z \in K, \neq x$, a necessary and sufficient condition for $z$ to lie in the inward set of $x$ is that the line segment connecting $x$ and $z$ meet $K$ in some point other than $x$.

A map $F: K \rightarrow X$ will be called "inward" if for all $x \in K, F(x)$ belongs to the inward set of $x$. The class of inward maps clearly includes the class of inner maps.

Similarly, the "outward set" of $x$ with respect to $K$ will mean the set of points $(1-\alpha) x+\alpha y$ for $y \in K$ and (N.B.) $\alpha \leqq 0$, and $F$ will be called outward if $F(x)$ always belongs to the outward set of $x$.

The "weakly inward" and "weakly outward" sets of a point $x$ will be defined as the closures of the inward and outward sets of $x$, respectively. "Weakly inward" and "weakly outward" maps will mean maps taking every $x$ to a member of the appropriate set.

We note that if $F$ is a (weakly) inward map, then the map $x \mapsto 2 x-F(x)$ is (weakly) outward, and conversely. Also, $x$ is a fixed point of one map if and only if it is a fixed point of the other. Hence fixed-point results for (weakly) inward maps

Received by the editors September 19, 1966.

( ${ }^{1}$ The results presented in this paper are strengthenings of some of the results obtained by the first author in his Ph.D. thesis at the University of California at Los Angeles. The first author held a National Aeronautics and Space Administration Graduate Fellowship and the second a National Science Foundation Graduate Fellowship while the work was done. 
are equivalent to such results for (weakly) outward maps. We shall derive our results by considering maps of the former type.

2. The strictly-convex-normed case. Suppose that the $X$ considered above is a strictly convex normed linear space. Then to every point $y \in X$ there corresponds a unique point $N_{K}(y) \in K$ whose distance to $y$ is minimal. The function $N_{K}$ so defined is a continuous retraction of $X$ onto $K$.

Given $x \in K$, let us define the "normal-outward set of $x$ " to be the set of points $y \neq x$ such that $N_{K}(y)=x$.

Given any $x \in K$, the weakly-inward set of $x$ and the normal-outward set of $x$ are disjoint. To show this, we must find, given $y$ in the normal-outward set of $x$, a neighborhood of $y$ containing no members of the inward set of $x$. We claim that the open ball of radius $\|x-y\|$ about $y$ has this property. For, given $z$ in this ball, all points of the segment joining $z$ and $x$, other than $x$ itself, belong to this ball, and hence are nearer to $y$ than $x$ is. Since $x$ is the point of $K$ nearest to $y$, no point of this segment can belong to $K$, hence $z$ is not in the inward set of $x$.

Let us call a map $F: K \rightarrow X$ "nowhere normal-outward" if $F(x)$ belongs to the normal-outward set of $x$ for no $x$. It is clear from the above that the class of maps so defined includes the weakly inward maps.

THEOREM 2.1. Let $X$ be a strictly convex normed linear space, $K$ a compact convex subset of $X$, and $F$ a nowhere normal-outward map from $K$ into $X$. Then $F$ has a fixed point.

Proof. $N_{K} F$ is a continuous map of $K$ into itself. Hence by the Schauder-Tychonoff theorem, there exists $x$ in $K$ such that $N_{K} F(x)=x$. Looking at the definition of the "normal-outward set of $x$ ", we see that $F(x)$ must belong to that set unless $F(x)=x$. Hence $F(x)=x$.

3. The case $X=\boldsymbol{R}^{\infty}$, and a Fibering Lemma. Let $\boldsymbol{R}^{\infty}$ designate the space of all sequences $x=\left(x_{1}, x_{2}, \ldots\right)$ of real numbers, with the product topology. Let $p_{i}: \boldsymbol{R}^{\infty} \rightarrow \boldsymbol{R}$ be the $i$ th-coordinate map.

LEMMA 3.1. Let $K$ be a compact convex subset of $\boldsymbol{R}^{\infty}$, and $F$ a weakly inward map from $K$ into $\boldsymbol{R}^{\infty}$. Then $F$ has a fixed point.

Proof. Suppose $F$ has no fixed point. Then the sets $U_{i}=\left\{x \in K \mid p_{i} F(x) \neq p_{i}(x)\right\}$ cover $K$, hence some finite number of them-say $U_{1}, \ldots, U_{n}-\operatorname{cover} K$. Thus the function $\sup _{i=1, \ldots, n}\left|p_{i} F(x)-p_{i}(x)\right|$ is nowhere zero, hence is bounded away from zero. We can clearly assume it is everywhere $\geqq 1$.

By the assumption that $F$ is weakly inward, we can, for every $x \in K$, find $y \in K$ and $\alpha \geqq 0$, such that the first $n$ coordinates of $u(x)=(1-\alpha) x+\alpha y$ differ from those of $F(x)$ by less than $1 / 2^{n}$. Now it is clear that the $y$ and $\alpha$ chosen for a given $x$ will work for all $x^{\prime}$ in a neighborhood of $x$ in $K$. Hence by compactness of $K$, we can handle all points of $K$ by choosing $y$ and $\alpha$ from some finite set; $u$ can thus be 
chosen as a function which, though not necessarily continuous, will take on all its values in a compact set. Hence for each $i>0$, we can find a real number $B_{i}$ such that $\forall x \in K,\left|p_{i}(x)\right|<B_{i},\left|p_{i} F(x)\right|<B_{i}$, and $\left|p_{i}(F(x)-u(x))\right|<B_{i}$. Multiplication of each coordinate by an independent constant is a linear homeomorphism on $\boldsymbol{R}^{\infty}$, hence preserves all the structure we are considering. Consequently, we may assume $B_{i} \leqq 2^{-i}$ for all $i>n$. (We have already put conditions on the first $n$ coordinates.)

Now let $H$ designate the space of all $L_{2}$ (square-summable) sequences of real numbers under the $L_{2}$ norm-a vector subspace of $\boldsymbol{R}^{\infty}$, but with a stronger topology. Let $B$ be the set of all $x=\left(x_{1}, x_{2}, \ldots\right)$ such that $\forall i\left|x_{i}\right| \leqq B_{i} . B$ lies in $H$, and it is easily shown that the $\boldsymbol{R}^{\infty}$ and $H$-topologies agree on $B$. Hence $K$ is a compact convex subset of $H$ and $F$ is continuous in the topology of $H$.

We note that for all $x \in K, u(x)$ is at a distance less than 1 from $F(x)$, since $\left|p_{i}(u(x)-F(x))\right|<1 / 2^{i}$ (for $i$ both $\leqq n$ and $>n$ ). On the other hand, $F(x)$ is at a distance at least 1 from $x$ (see first paragraph). Hence $u(x)$, a point on a ray drawn from $x$, through some other point of $K$, is nearer to $F(x)$ than $x$ is. Some point on the line segment between $x$ and $u(x)$ will both be closer to $F(x)$ than $x$ is and be in $K$. Hence $x$ is not the point of $K$ nearest $F(x)$. So $F$ is a nowhere normal-outward map without a fixed point, contradicting Theorem 2.1 .

We shall obtain our most general form of the fixed-point theorem from the above by the Fibering Lemma and the corollary below. (This is a strengthened form of the argument used in the Dunford-Schwartz lemma [1, Chapter V, 10.4]-the analogous step in the proof of the Schauder-Tychonoff theorem.) Note that our lemma merely requires $K$ to be Lindelöf (every open covering has a countable subcovering), though in the case we are interested in, it is compact.

LEMMA 3.2 (FIBERING LEMMA). Let $X$ be a topological vector space whose topology is induced by linear functionals, let $K$ be a Lindelöf subset of $X$, and let $F: K \rightarrow X$ be a continuous map. Then, given any countable family $G_{0}$ of continuous linear functionals on $X$, there is a continuous linear map $p: X \rightarrow \boldsymbol{R}^{\infty}$, and a continuous map $F^{\prime}: p(K) \rightarrow \boldsymbol{R}^{\infty}$ such that:

(1) $F^{\prime} p=p F$

(2) For each $f \in G_{0}$ there exists an $f^{\prime}: \boldsymbol{R}^{\infty} \rightarrow \boldsymbol{R}$ such that $f=f^{\prime} p$.

Proof. We shall first show that any continuous real-valued function $g$ on $K$ is (in a sense to be made clear) "continuously determined" by a countable family of continuous linear functionals of $X$.

Given $g$ we can, by the Lindelöf assumption, find for each $\varepsilon>0$ a countable covering of $K$ by open sets $\left(U_{\alpha}\right)_{\alpha \in A_{\varepsilon}}$, such that for each $\alpha$ in the index-set $A_{\varepsilon}$, and $x, x^{\prime} \in U_{\alpha},\left|g(x)-g\left(x^{\prime}\right)\right|<\varepsilon$. By the assumption on the topology of $X$, each $U_{\alpha}$ can be assumed of the form

$$
\left\{x \in K \mid f_{\alpha 1}(x) \in\left(a_{\alpha 1}, b_{\alpha 1}\right), \ldots, f_{\alpha n_{\alpha}}(x) \in\left(a_{\alpha n_{\alpha}}, b_{\alpha n_{\alpha}}\right)\right\}
$$

where the $f_{\alpha i}$ are continuous linear functionals on $X$, and the $a_{\alpha i}<b_{\alpha i}$ are real numbers. 
Let $A$ be the union of index-sets $A_{1} \cup A_{1 / 2} \cup A_{1 / 4} \cup \ldots$. Then it follows from our construction that $g$ is a continuous function of the family of maps $\left(f_{\alpha i}\right)_{\alpha \in A, i=1, \ldots, n_{\alpha}}$. I.e., $g$ can be written $g_{0} f$, where $f$ is the product map of the $f_{\alpha i}$, sending $X$ into $\boldsymbol{R}^{\left\{(\alpha, i) \mid \alpha \in A, i \leqq n_{\alpha}\right\}}$, and $g_{0}$ is a continuous function on $f(K)$, as we desired.

We are given a set of functionals $G_{0}$; for $j>0$ let us, inductively, make $G_{j}$ a countable family of linear functionals which, for every $f \in G_{j-1}$, "continuously determines" $f F$. Let $G=\bigcup_{j=0}^{\infty} G_{j}$, which we can reindex $\left(f_{i}\right)_{i=1,2, \ldots}$, since it is countable. (If $G$ is finite, we let $f_{i}=0$ for large $i$.) Let $p: X \rightarrow \boldsymbol{R}^{\infty}$ be the product of this family of maps. It is clear from our construction that for all $i, f_{i} F$ is continuous determined by $p$, thus $p F$ is continuously determined by $p$, i.e., we can write $p F=F^{\prime} p$, where $F^{\prime}$ is continuous. On the other hand, for every $f \in G_{0}, f$ will equal some $f_{i}$; letting $f^{\prime}$ be the $i$ th projection map we have $f=f^{\prime} p$.

COROllary 3.3. Lemma 3.2 still holds if the hypothesis "the topology of $X$ is determined by linear functionals," is replaced by "linear functionals distinguish points of $X$, and $K$ has compact closure."

Proof. Let $X^{\prime}$ designate $X$ with the topology induced by the continuous linear functionals. Since the closure of $K$ is compact in $X$, the closure of $K$, and hence $K$ itself, has the same topology in $X^{\prime}$ as in $X$. Hence continuous maps on $K$ remain continuous in the $X^{\prime}$ topology; and we get our results by applying Lemma 3.3 in $X^{\prime}$.

\section{The general fixed-point theorems.}

THEOREM 4.1. Let $X$ be a topological vector space such that continuous linear functionals distinguish points. Let $K$ be a compact convex subset of $X$, and $F: K \rightarrow X a$ weakly inward map. Then $F$ has a fixed point.

Proof. Given any continuous linear functional $f$ on $X$ let $S_{f}$ be the set of $x \in K$ such that $f F(x)=f(x)$. We claim that any finite intersection of the sets $S_{f}$ is nonempty.

Indeed, given any finite set, $G_{0}$, of such functionals, we apply Corollary 3.3, getting maps $p: X \rightarrow \boldsymbol{R}^{\infty}$ and $F^{\prime}: p(K) \rightarrow \boldsymbol{R}^{\infty}$. The set $p(K)$ is compact and convex. Further, $F^{\prime}$ is weakly inward, for it is easy to see that the property of lying in the weakly inward set of a point is preserved under continuous linear maps of the vector space.

So by Lemma 3.1, $F^{\prime}$ has a fixed point $p(z), z \in X$. We claim that $z \in \bigcap_{a_{0}} S_{f}$. For given any $f \in G_{0}$, we compute

$$
f F(z)=f^{\prime} p F(z)=f^{\prime} F^{\prime} p(z)=f^{\prime} p(z)=f(z) .
$$

It follows from the compactness of $K$ that the intersection of the sets $S_{f}$ over all linear functionals $f$ is nonempty. Clearly, a point in this intersection is a fixed point of $F$. 


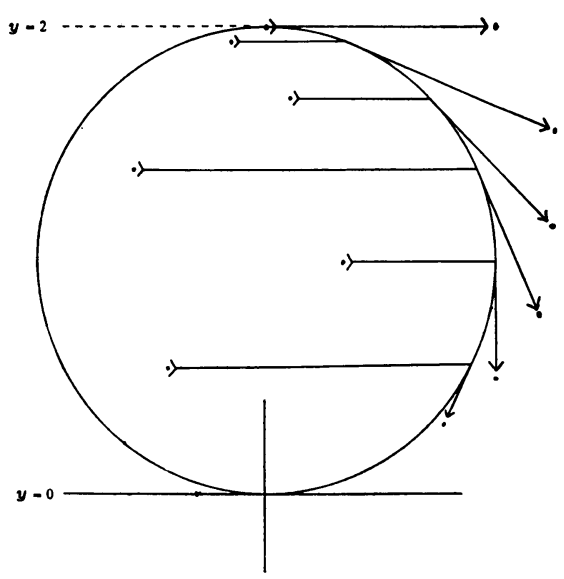

FIGURE 1

Given a map $F$ on a compact convex set $K$ in a topological space $X$, let us call a coset $C$ of a closed subspace of $X$ a nonempty section of $K$ if $C \cap K \neq \varnothing$; and let us call it an invariant section if $F(C \cap K) \subset C$. The proof in Dunford-Schwartz [1] of the general case of the Schauder-Tychonoff theorem makes use of the fact that the inverse image of a fixed point under a fibering is a nonempty invariant section, and that if $C$ is a nonempty invariant section and $F$ is an inner map, then $(F \mid K \cap C):(K \cap C) \rightarrow C$ is again an inner map. (Zorn's lemma is then used.) The latter result also holds for inward maps, but not for weakly inward maps! In fact, for $F$ weakly inward, a nonempty invariant section need not contain a fixed point. For example, let $K$ be the disc $x^{2}+(y-1)^{2} \leqq 1$ in the $x y$-plane, and let $F$ send the point $(x, y)$ to the end of the clockwise segment of length $y / 2$ tangent to $K$ at the point $\left(\left(1-(y-1)^{2}\right)^{1 / 2}, y\right)$. Then $y=0$ and $y=2$ are both invariant sections, but only $y=0$ has a fixed point. (See Figure 1.)

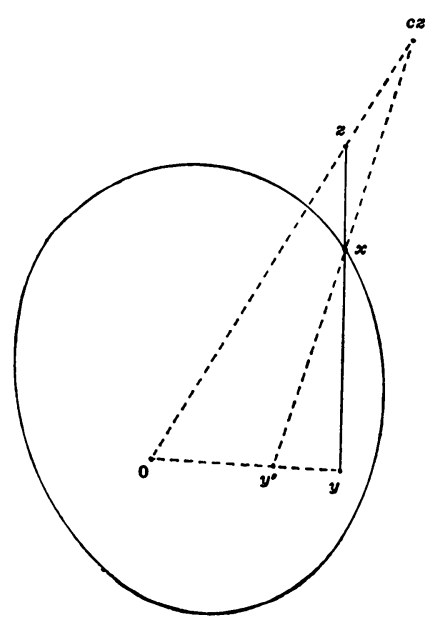

FIGURE 2 
It was this observation that forced us to look, not at sets based on an arbitrary choice, but sets such as the $S_{f}$ 's which cannot exclude any potential fixed points.

Lemma 4.2. Suppose a compact convex subset $K$ of a topological vector space $X$ contains the point 0 . Then the outward set of any point $x$ is closed under multiplication by constants $c>1$. Hence so is the weakly outward set.

(Proof. See Figure 2, where $z=(1-\alpha) x+\alpha y(\alpha<0)$ is an arbitrary point of the outward set of $x$. The reader can easily supply the numerical argument, getting $c z$ in the form $\left(1-\alpha^{\prime}\right) x+\alpha^{\prime} y^{\prime}$.

THEOREM 4.3. Let $X$ be a topological vector space such that continuous linear functionals distinguish points. Let $K$ be a compact convex subset of $X$, and $F: K \rightarrow X$ a weakly outward map. Then:

(1) F has a fixed point,

(2) $F(K) \supset K$.

Proof. (1) is clear from Theorem 4.1 and our original discussion of the relationship between "inwardness" and "outwardness" conditions.

To show (2), let us suppose the contrary. Clearly, we can assume that 0 is a point of $K-F(K)$. The complement $U$ of $F(K)$ is a neighborhood of 0 , so we can choose $c>1$ such that $c U \supset K$. Then $c F(K)$ is disjoint from $K$, and so the map $c F$ can have no fixed points. But, by Lemma 4.2 it is clear that $c F$ is weakly outward. Contradiction.

\section{REFERENCES}

1. Nelson Dunford and Jacob T. Schwartz, Linear operators, Part I, Interscience, New York, 1964.

2. A. Tychonoff, Ein Fixpunktsatz, Math. Ann. (1935), 767-776.

UNIVERSITY OF CALIFORNIA, Los Angeles, California

HARVARD UNIVERSITY,

CAMbridge, Massachusetts 